\title{
An Independent Review of Existing Total Solar Irradiance Records
}

\author{
Pia Zacharias
}

Received: 15 April 2013/Accepted: 14 May 2014/Published online: 14 June 2014

(C) Springer Science+Business Media Dordrecht 2014

\begin{abstract}
With the advent of space-based total solar irradiance (TSI) observations about 35 years ago, researchers' understanding of solar variability and its causes has greatly improved. Controversies regarding the cross-calibration of the data from various TSI instruments have resulted in many different TSI composite time series. These composites agree well with each other on timescales ranging from days to years, but due to the limited stability of the instruments contributing to the composites, their quality is not yet sufficient to unambiguously detect possible changes between subsequent cycle minima. In this paper, the construction of the three most prominent TSI composite time series and the underlying TSI models is addressed. The difficulties associated with the cross-calibration of the data are considered, and the viewpoints of the different groups involved in the development of the composites are discussed.
\end{abstract}

Keywords Total solar irradiance $\cdot$ Solar variability $\cdot$ Solar activity

\section{Introduction}

Total solar irradiance (TSI), a measure of the spectrally and spatially integrated radiant energy from the Sun at the mean Earth distance of $1 \mathrm{AU}$, is the dominant energy source influencing the Earth's climate system. A century of ground-based solar irradiance observations failed to reveal unambiguous variations associated with solar activity because of the noise in the observations induced by the Earth's atmosphere which scatters, reflects and absorbs solar photons. The solar energy flux was thus originally assumed to be a

\author{
P. Zacharias $(\bowtie)$ \\ International Space Science Institute, Hallerstrasse 6, 3012 Bern, Switzerland \\ e-mail: pia.zacharias@astro.uio.no \\ P. Zacharias \\ Institute of Theoretical Astrophysics, University of Oslo, Blindern, P.O. Box 1029, 0315 Oslo, Norway
}


Table 1 List of space-borne TSI instruments used for construction of the various TSI composites

\begin{tabular}{llll}
\hline Instrument & Mission & Observation period & References \\
\hline HF & NIMBUS7 & Nov 1978-Jan 1993 & Hoyt et al. (1992) \\
ACRIM-I & SMM & Feb 1980-Jun 1989 & Willson (1984) \\
ERBE & ERBS & Oct 1984-Oct 2005 & Lee III et al. (1995) \\
ACRIM-II & UARS & Oct 1991-Sept 2001 & Willson (1994) \\
VIRGO/DIARAD & SOHO & Feb 1996-present & Fröhlich et al. (1997) \\
VIRGO/PMO6 & SOHO & Feb 1996-present & Fröhlich et al. (1997) \\
ACRIM-III & ACRIMSAT & May 2000-present & Willson (2001) \\
TIM & SORCE & Feb 2003-present & Kopp et al. (2003) \\
PREMOS & PICARD & Jun 2010-present & Schmutz et al. (2009) \\
\hline
\end{tabular}

${ }^{a}$ Not included in TSI composite, but used for comparison

constant and referred to as "solar constant" (e.g., Abbot 1958 and references therein). Only with the advent of space-based observations could the nature of solar irradiance variability be established (Willson et al. 1981).

TSI satellite observations using electrically calibrated radiometers started with the launch of Hickey-Frieden (HF) on Nimbus7 in November 1978. Up to now, TSI satellite observations cover 35 years of continuous data record, i.e., data of nearly three solar activity cycles. At least two different spacecraft have been monitoring TSI simultaneously since 1980. However, the individual instruments have operated for only limited intervals (see Table 1). Therefore, a combination of data from several different instruments is required to compile a continuous time series. Differences in absolute flux measurements of the radiometers as well as the instruments' change in time have raised difficulties in the cross-calibration of the data. Thus, the construction of a TSI time series has resulted in different TSI composites. The three most prominent examples are the PhysikalischMeteorologisches Observatorium Davos (PMOD) composite by Fröhlich and Lean (1998a), the Active Cavity Radiometer Irradiance Monitor (ACRIM) composite published by Willson (1997) and the Institut Royal Météorologique Belgique (IRMB) composite by Dewitte et al. (2004). ${ }^{1}$ These composites are shown in Fig. 1.

The three composites are constructed from the same original data, which are also shown in Fig. 1, but not all composites use all data (e.g., the ACRIM composite does not use VIRGO data, and the PMOD composite does not use data from ACRIM-III) and different individual datasets are applied for some periods. Also, different procedures are applied to correct for the sensitivity changes of the various radiometers. Until 1996, the time series of data from the HF instrument on Nimbus7, the ACRIM-I instrument on the Solar Maximum Mission (SMM) spacecraft and the ACRIM-II instrument on the Upper Atmosphere Research Satellite (UARS) are used. After 1996, the ACRIM composite continues to use ACRIM-II data supplemented by datasets from ACRIM-III on ACRIMSat, whereas the PMOD and IRMB composites use different combinations of data from the VIRGO instrument on the Solar Heliospheric Observatory (SoHO) spacecraft. Datasets from the more recently launched TIM/SORCE and PREMOS/PICARD instruments are mostly used for comparison, but data from TIM have been included in the IRMB composite.

1 Updated versions of the PMOD, ACRIM and IRMB composites can be found in Fröhlich (2009), Willson and Mordvinov (2003), and Mekaoui and Dewitte (2008). 

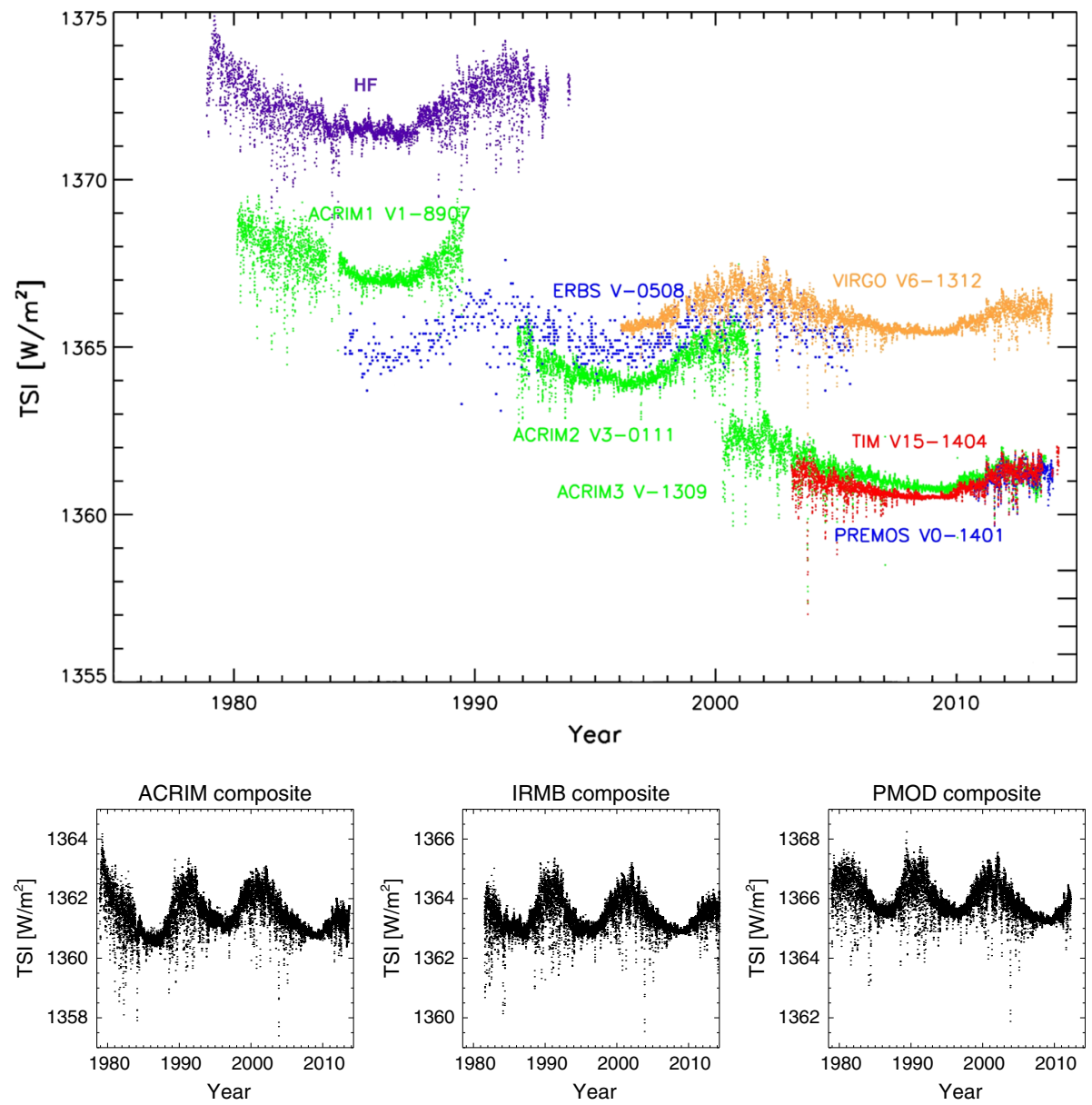

Fig. 1 Top panel: TSI records from radiometers on different space platforms: HF on Nimbus7, ACRIM-I on SMM, ERBE on ERBS, ACRIM-II on UARS, VIRGO on SOHO, ACRIM-III on ACRIM-Sat, TIM on SORCE and PREMOS on PICARD. The figure is an updated version of Fig. 1 from Kopp et al. (2012) (courtesy of G. Kopp, data source: http://spot.colorado.edu/ koppg/TSI/, status April 9, 2014). It has been slightly adapted to show only the instrument records discussed in this work. Bottom panels: The three most prominent TSI composites. The ACRIM composite (version 11/13, http://www.acrim.com/Data\% 20Products.htm) is shown on the left, the IRMB composite (Dewitte et al. 2004, Mekaoui and Dewitte 2008, data source: ftp://gerb.oma.be/steven/RMIB_TSI_composite/) is shown in the middle, and the PMOD composite (version d41_62_1204, data source: ftp://ftp.pmodwrc.ch/pub/Claus/IrradianceData/) is shown on the right

The largest TSI amplitude variations are associated with the appearance of photospheric features on the solar disk. The net effect of dark sunspots on TSI can lead to a drop of up to $0.25 \mathrm{~W} \mathrm{~m}^{-2}$, and bright faculae can cause a TSI increase of up to $\approx 0.1 \mathrm{~W} \mathrm{~m}^{-2}$ during their passage across the solar disk (Hudson et al. 1982, Willson 1984, Fröhlich 2013). However, much larger drops in TSI can be produced by large sunspots groups, and likewise faculae can show much larger increases, e.g., a drop of $4.6 \mathrm{~W} \mathrm{~m}^{-2}$ has been observed during the October 2003 storm period (Woods et al. 2004). In principle, the origin of these 
short-term variations on rotational timescales is well understood and can be explained reliably by TSI models (Foukal and Lean 1988). This is also true for the 11-year modulation by the Sun's activity cycle. The $\sim 0.1 \%$ modulation of TSI between solar activity minimum and maximum in recent decades is now a well-established feature (Willson and Hudson 1991).

However, in order to study a possible long-term (secular) modulation of TSI, e.g., the trend between successive activity minima, careful cross-calibration of the various experiments is required. Modern instruments require an absolute accuracy of one-tenth of the solar cycle variability and repeatability (relative precision per year) of at least one-tenth of the accuracy (Lean and Woods 2010). For the combination of data from different satellites, corrections for absolute level calibration, degradation and switch-off periods of each instrument need to be taken into account. Many of these issues, which will be outlined and discussed below, are still a matter of active debate and have prevented the TSI community from coming up with a conclusive TSI composite so far. This review aims at summarizing the most important arguments put forth by the various representatives and tries to give an independent view on the approaches favored by different authors on assessing the accuracies and uncertainties in the TSI data record.

\section{Functional Principle of Active Cavity Radiometers}

All of the instruments considered in this review measure TSI in space by applying the same fundamental principle, that of an active cavity radiometer (e.g., Fröhlich et al. 1995; Butler et al. 2008). A beam of sunlight is absorbed by a black, metallic, thermally isolated cavity after having passed through a circular aperture, typically a few millimeters in diameter. The temperature difference between the absorbing cavity and a non-illuminated cavity is actively controlled. The additional electrical heater power required to maintain this temperature difference when closing the shutter, i.e., blocking the sunlight, is measured. Except for small corrections, the ratio of this power measurement to the aperture area gives the value for TSI at the defining aperture. Information on the spacecraft ephemeris is then used to convert this value to a Sun-instrument distance of 1 AU.

Inaccuracies in the measurement of the entrance aperture area could, in principle, cause the observed differences in TSI measurements. However, laboratory measurements with existing flight units have shown that intra-instrument scatter is the largest source of uncertainty (e.g., Kopp et al. 2012), with apertures area effects being relatively minor (Butler et al. 2008). The lack of equivalence of optical and electrical power and the need for corrections to account for aperture diffraction and Sun-Earth distance represent additional sources of uncertainty. Long-term precision is affected by changes in the optical properties of the cavity surface, drifts in the electrical components and changes of the thermal environment (Lean and Woods 2010).

The exposure of the radiometers to the strong solar radiation in space leads to degradation effects and sensitivity changes over time (Fröhlich et al. 1995). These timedependent degradation effects can be determined by comparison with a less exposed backup radiometer of the same type. Unfortunately, of the early TSI instruments, only the ACRIM and VIRGO radiometers have backups, whereas the Nimbus7/HF and ERBS/ ERBE instruments lack such a backup radiometer. As outlined in the following, this has resulted in many disputes between various instrument teams on how to correct for the degradation effects of these two instruments, which are critical for spanning data gaps between other instruments able to track degradation changes. 
An early increase in sensitivity has been identified for the PMO6V radiometers on VIRGO/SoHO (Anklin et al. 1998). Fröhlich (2006) argues that this effect is due to the blackening of the primary aperture by solar UV radiation that leads to an increase in temperature of the innermost aperture and simulates increased sensitivity by extra IR radiation emitted into the cavity. He claims that the effect is important for all radiometers which have their primary aperture directly in front of the cavity and have apertures with a small cylindrical part, such as the HF, ACRIM, ERBS and PMO6 radiometers, but not for the DIARAD. Such a statement requires additional proof and a verification by the respective instrument teams. However, it is interesting to note that the PREMOS instrument, which is nearly identical in design to the PMO6 on VIRGO, sees a very similar large early increase, as presented by Schmutz et al. during the ISSI Team's first meeting on “An Assessment of the Accuracies and Uncertainties in the Total Solar Irradiance Climate Data Record" in March $2012^{2}$ and in Schmutz et al. (2013). Based on similarities between the Nimbus7/HF and PMO6V/VIRGO instruments (HF has the same geometry of the cavity as the PMO6V type instruments and uses the same black paint), an early increase of sensitivity similar to the one found for PMO6V has been applied for the HF radiometer by Fröhlich and his coworkers (Fröhlich and Lean 1998a; Fröhlich 2006). However, this extrapolation of similarities between PMO6V and HF has been criticized by Willson and Mordvinov (2003) who argue that significant differences between the instruments exist and that the instruments were observing during opposite solar cycle phases.

In addition, a non-exposure-dependent degradation effect has been shown to characterize the sensitivity changes of the PMO6V radiometers (Anklin et al. 1998). This effect can be measured by direct comparison of two different types of radiometers, after they have been individually corrected for the exposure-dependent part (Fröhlich 2003). The characteristics of this effect are best described by an exponential behavior, but the exact causes are not known (Fröhlich 2009). The major advantage of having two different kinds of radiometers on the same instrument, such as the DIARAD and PMO6V radiometers on the VIRGO instrument, is that the corrected data from both radiometers can be used to check for the reliability of the individual corrections (Fröhlich et al. 1995). Major differences between the DIARAD and PMO6V radiometers are the arrangements of the compensating cavities as well as their shapes and coatings, which results in different degradation effects for both instruments (Fröhlich et al. 1995; Fröhlich 2003; Dewitte et al. 2004).

Newer spaceflight instruments, such as PREMOS (Schmutz et al. 2009) and TIM (Kopp et al. 2003), have benefited from these discussions and previous calibration studies. TIM is the only TSI instrument that uses a reverse order of the two beam-limiting apertures in front of the cavity by placing the defining aperture (the smaller one of the two which determines the area over which the sunlight is collected) in front of the view-limiting aperture (see, e.g., Butler et al. 2008 for details). In the classical arrangement used by most instruments the larger view-limiting aperture is placed in front of the defining aperture which allows more light to enter the instrument interior potentially leading to higher scatter, since only a fraction of the rays entering the view-limiting aperture also enter the defining aperture. The other rays are captured by the baffles located between the view-limiting and defining apertures. A small fraction, however, will be scattered off the baffles or the solar-illuminated view-limiting aperture and enter the defining aperture, leading to a contribution to the directly illuminating beam. This effect, if not entirely corrected for, would then lead to an overestimation of the solar irradiance. As discussed in Sect. 3, the $5 \mathrm{~W} \mathrm{~m}^{-2}$ difference between previous TSI measurements and those of TIM opened new views on the role of

\footnotetext{
${ }^{2}$ http://www.issibern.ch/teams/solarirradiance/.
} 
scattering effects and led to new instrument calibration and design studies (Butler et al. 2008). TIM also uses a new algorithm to deduce the power measurement from the raw data (see Kopp et al. 2003 for details). While all other instruments do this analysis in the time domain, TIM data are analyzed in the frequency domain. This phase-sensitive detection scheme reduces the sensitivity to thermal drifts, $1 / \mathrm{f}$ noise and thermal emission from the heat sink (which will be out-of-phase with the shutter).

The PREMOS instrument is characterized by its end-to-end calibration in vacuum against an SI-traceable irradiance reference. These vacuum calibrations avoid the complications of air conduction and convection and more appropriately simulate on-orbit performance. More details on the instrument calibration can be found in Schmutz et al. (2009). PREMOS was the first instrument to be launched after such a calibration. Its contribution to the determination of an absolute TSI value is discussed in Sect. 3.

\section{Determination of the Absolute TSI Value}

The TSI values and their relative variation can, in principle, be normalized to any reference level, and the determination of an absolute TSI value seems to be merely a side issue in this context. This is also true for many climate models that use the TSI variability rather than an absolute TSI value as input parameter. However, absolute accuracy has recently been shown to be important for estimates of Earth's energy balance (Wild et al. 2013). Also, it is, to a large extent, the difficulty in measuring the absolute fluxes and differences among the individual instruments that make the inter-calibration of individual records so difficult. Therefore, the latest developments in the determination of an absolute TSI value are considered to be a valuable contribution to the overall picture on TSI.

In the 1990s, it was generally considered that measurements were converging to an absolute TSI value of $(1366 \pm 1) \mathrm{W} \mathrm{m}^{-2}$ (Crommelynck et al. 1995; Fröhlich and Lean 1998a). However, after data from TIM/SORCE (launched in 2003) had become available, a new absolute TSI value was published that was approximately $5 \mathrm{~W} \mathrm{~m}^{-2}$ lower compared to previous measurements (Kopp et al. 2003, 2005; Kopp and Lean 2011). Lately, Kopp and Lean (2011) and Kopp et al. (2012) have favored a TSI value of $(1360.8 \pm 0.5) \mathrm{W} \mathrm{m}^{-2}$ as being the best representative value of solar minimum. The lower TSI value was thought to be a likely consequence of the reverse arrangement of the TIM instrument's apertures compared to the classical radiometers. The classical arrangement may increase the susceptibility to stray light from the view-limiting aperture and radiation reflected back into the cavity. In principle, these effects are accounted for in the characterization of the instruments. However, they seemed to have been underestimated (Fröhlich 2013; Kopp and Lean 2011). To overcome the discrepancy between different TSI instruments, a workshop was held at the National Institute of Standards and Technology (NIST) in 2005. Neither TIM's new approach to data processing that is based on a phase-sensitive detection scheme nor the cavity coating and design differences between the classical active cavity radiometers and TIM were identified to be responsible for the $0.35 \%$ TSI scale difference. Since modeling the scatter within the instruments was known to be unreliable, laboratory measurements with the laboratory versions of all existing flight units were recommended at the NIST workshop (Butler et al. 2008).

The TSI Radiometer Facility (TRF) at LASP has been designed for such an overall assessment of stray light and other effects by comparing the measurements with a cryogenic reference radiometer (Kopp et al. 2007). Kopp et al. (2012) report on TRF tests of a ground-based SORCE/TIM, a PMO6 radiometer representative of the SoHO/VIRGO, a 
ground representative of the ACRIMSat/ACRIM-III and a flight and a ground-based PICARD/PREMOS. These tests indicated that the causes of the offsets between the ACRIMIII, PREMOS, VIRGO and TIM TSI instruments are principally internal instrument scatter for ACRIM-III and a combination of scatter and measured optical power for VIRGO and PREMOS.

Finally, PREMOS/PICARD measurements helped to resolve the discrepancy between VIRGO and TIM in the actual TSI value. First light measurements of PREMOS obtained in July 2010 yielded a solar constant of $(1360.9 \pm 0.4) \mathrm{W} \mathrm{m}^{-2}$ (Schmutz et al. 2012). It was shown that the stray light contribution for instruments having a view-limiting aperture in front and a smaller precision aperture behind, such as the PMO6 and ACRIM type instruments, had been severely underestimated in the past. By applying a similar stray light correction to the VIRGO flight instruments to the one determined for a spare VIRGO instrument of type PMO6V at the TRF, the VIRGO and PREMOS values were reduced by $4 \mathrm{~W} \mathrm{~m}^{-2}$, thus yielding a consistent TSI value for TIM/SORCE, VIRGO/SOHO (corrected) and PREMOS/PICARD.

\section{Construction of the Various TSI Composites}

A critical issue for the construction of the TSI composites is the 2-year data gap between the end of SMM with the ACRIM-I instrument in mid-1989 and the start of UARS with the ACRIM-II instrument in late 1991, the so-called ACRIM gap. Two datasets, the Nimbus7/ $\mathrm{HF}$ and the ERBS/ERBE datasets, are available during that time. However, both instruments were designed to provide TSI results for Earth Radiation Budget (ERB) investigations and not for TSI monitoring, which requires much higher precision, accuracy and traceability. The HF and the ERBE instrument both lack a spare radiometer to monitor effects of degradation and, in addition, show opposite trends in TSI during the ACRIM gap (e.g., Willson and Mordvinov 2003). The Nimbus7/HF data show an increase of the TSI with time, whereas the ERBS/ERBE data show a significant decrease of TSI with time during that period. There are two possibilities to explain the different trends in the HF and ERBS datasets. An increase of ERBS/ERBE degradation relative to Nimbus7/HF could explain the decrease of the ERBE/ERBS TSI data during the time of the ACRIM gap and is the approach favored by the ACRIM group. On the other hand, an increase in the sensitivity of the HF sensor could explain the increase of the Nimbus7/HF TSI data during that phase and is the approach favored by the PMOD group.

In addition to the difficulties concerning the choice of a suitable instrument record during the ACRIM gap, further complications arise due to the possible existence of glitches in the instruments' data records, such as jumps in the data of the HF instrument during that phase or jumps in the ACRIM-I dataset after a switch-off of the instrument in April 1984. Another major point of discussion is the impact of the so-called SMM 'spin mode phase' (December 1980-April 1984) on the long-term stability of the ACRIM-I instrument. Details of all these issues and their effect on secular trends of the TSI composites are outlined below.

\subsection{Jumps and Glitches in the HF Data Record}

The existence of jumps and glitches in the HF data was first addressed by Hoyt et al. (1992) who compared TSI data of the HF and ERBE instruments and applied models based on 
direct photometric observations of sunspots and faculae for comparison. Lee et al. (1995) identified an upward shift in the irradiance scale of the HF instrument of $0.4 \mathrm{~W} \mathrm{~m}^{-2}$ for the interval September/October 1989-April 1990 and an additional upward shift of $0.4 \mathrm{~W} \mathrm{~m}^{-2}$ for the interval May 1990-January 1993. Chapman et al. (1996) found an upward shift of $0.31 \mathrm{~W} \mathrm{~m}^{-2}$ in the HF data for the interval from September 1989-May 1990 followed by an additional upward shift of $0.37 \mathrm{~W} \mathrm{~m}^{-2}$ for the interval from May 1990-December 1993.

In the original version of the PMOD composite, Fröhlich and Lean (1998a) include a -0.26 and $-0.32 \mathrm{~W} \mathrm{~m}^{-2}$ downward correction of the Nimbus7/HF data on October 1, 1989, and May 8, 1990, referring to the work of Lee et al. (1995) and Chapman et al. (1996) for their adjustments. Later on, Fröhlich and Lean (2002) suggested that the two glitches may reflect the continuation of a general increase in sensitivity of the HF radiometer that had already been identified by comparison with ACRIM-I earlier in the record (between 1980 and 1984). Consequently, the authors assumed continuous, rather than isolated, HF sensitivity changes, in order to account for the difficulty of identifying the glitches as local events, such as changes in the orientation of the spacecraft (Fröhlich and Lean 2002). The combination of a slip on September 29, 1989, after a switch-off of the HF instrument and a linear trend was implemented in the PMOD composite yielding a total change of $-0.84 \mathrm{~W} \mathrm{~m}^{-2}$ during the gap (Fröhlich 2004). In a follow-up paper, the corrections for the entire observation period of the HF instrument (Novemver 1978-January 1993) were determined at once resulting in a new HF time series (Fröhlich 2006). This time series will be further discussed in Sect. 4.3.

The magnitude of the step in the HF data is crucial, since it constitutes a major difference between the PMOD and ACRIM composite and thus indicates whether or not there exists a long-term change of TSI. The corrections for glitches in the Nimbus7/HF data during the ACRIM gap incorporated in the PMOD composite have been criticized repeatedly by the advocates of the ACRIM composite (e.g., Willson and Mordvinov 2003; Scafetta and Willson 2009; Scafetta 2010). In particular, Willson and Mordvinov (2003) criticize the approach by Fröhlich of modifying the original results published by the ACRIM-I and HF science teams that according to Willson most accurately represent the instruments' performances. One of the main arguments that Scafetta holds against the HF corrections that Fröhlich applies is a private letter to Scafetta published in Scafetta (2010), in which D. Hoyt, the Nimbus7/HF principal investigator, remarks that he could not identify any problems with the HF instrument in September 1989. Hoyt et al. (1992) do not discuss the time period in question, but they do acknowledge that there were problems with the data in earlier periods, e.g., a sudden change in the noise level in 1980 or an apparent calibration change in September 1987 that appears similar to the presumed change in September 1989. Possible reasons for the 1987 calibration change have been proposed by Hoyt et al. (1992), such as a change in the electronics of the radiometer, but the ultimate source was not identified. The line of argument that the change occurred was essentially very similar to that of Lee et al. (1995) for the 1989 change, i.e., comparison with other data. Based on the published results, it can therefore not be excluded that another calibration change took place in 1987. However, the assumption that such an event occurred requires further proof. Since this constitutes a major point of discussion, an official comment by the HF instrument specialists would be desirable.

By assuming a continuous change of sensitivity of the HF instrument rather than isolated changes, Fröhlich and Lean (2002) found a way to circumvent the problem of identifying glitches that are not related to any obvious changes of the respective instrument. Nevertheless, this approach simply includes modifications of the original data, such 
as, e.g., corrections for the jump in September 1989, as well as the questionable adjustments of the early HF TSI values (1979-1980) and lacks a satisfying discussion of the physical causes leading to these issues.

Regardless of that, there is a major weak point in the discussion of Scafetta and Willson (2009), who have used one of the SATIRE models (see Sect. 5) to demonstrate that it supports the ACRIM composite. However, the authors of that model clearly state that the applied model is not suited for such an analysis (Krivova et al. 2009). By employing a more appropriate model in exactly the same way as proposed by Scafetta and Willson (2009), Krivova et al. (2009) were able to show their model is actually consistent with a deeper minimum in 1996 compared to 1986 and thus favors the PMOD composite.

\subsection{SMM Spin Mode Phase}

Between December 1980 and April 1984, the solar pointing system of the SMM satellite failed. The satellite was placed in a new operational mode in order to take advantage of the limited solar pointing capability. This resulted in a reduced quantity and quality of the ACRIM-I data during the so-called SMM 'spin stabilized mode' (see, e.g., Willson 1984 ${ }^{3}$ ).

For the construction of the PMOD composite, Fröhlich and Lean (1998a) assume that essentially all of ACRIM-I's degradation happened prior to the repair of SMM in April 1984. On the other hand, Willson and Mordvinov (2003) consider the degradation corrections of ACRIM-I data applied in the PMOD composite to be incorrect, since they are based on wrong assumptions regarding the cumulative solar exposure of its sensors during SMM's 'spin mode'. They refer to ACRIM-I's threefold redundant degradation self-calibration capability that provides a precise characterization of sensor degradation before and after the SMM 'spin mode' phase (Willson and Hudson 1991).

Nevertheless, Fröhlich (2006) argues that degradation of the ACRIM-I and HF sensors proceeded in a similar way for both instruments. Thus, similar degradation corrections can be applied for both ACRIM-I and HF based on similarities between the HF and the PMO6V radiometers on VIRGO/SoHO. The degradation of ACRIM-I during its first year of operation is therefore corrected for the effect of the shorter exposure time during the 'spin mode', although no unambiguous investigations justifying these modifications exist. However, the authors must be granted the fact that there are no realistic ways of recalibrating the old flight instruments nowadays, and thus, ideal calibrations are not feasible.

\subsection{Long-Term Trends in the PMOD and ACRIM Composites}

In the first version of the PMOD composite, Fröhlich and Lean (1998a), (1998b) introduced corrections for the HF degradation based on early results from PMO6V on VIRGO/ SoHO as well as for the ACRIM-I degradation during the SMM 'spin mode phase' (see Sects. 4.1 and 4.2). Corrections during the ACRIM gap were determined by comparison with ERBS/ERBE and a proxy model (Fröhlich 2000). This choice of data results in similar levels of solar irradiance during the solar activity minima in 1986 and 1996 (Fröhlich and Lean 1998a).

Willson and coworkers argue that the PMOD trend is an artifact of the effect of accelerated degradation of the ERBS/ERBE radiometer during the instrument's first

\footnotetext{
${ }^{3}$ Repair of the SMM pointing capability by the Space Shuttle in 1984 restored the high quality results available during normal operation in 1980 .
} 
exposure to the high UV flux during the rising activity phase of solar cycle 22 . In the ACRIM composite, the ACRIM-I/ACRIM-II ratio is derived from the comparison with Nimbus7/HF data using the original dataset published by the HF \& ACRIM-I science teams. This results in an TSI increase of $0.05 \%$-per-decade between consecutive solar cycle minima (Willson and Mordvinov 2003).

From 2006 on, Fröhlich applies a new HF time series for the period November 1978January 1993 which he refers to as "internally consistent" (Fröhlich 2006). This time series is composed of corrected HF data (November 1978-February 1980), corrected ACRIM-I data (February 1980-June 1989) and ERBS data (June 1989-January 1993). Corrections applied to the ACRIM-I data include a correction term for the exposuredependent increase of early sensitivity based on similarities between the ACRIM-I instrument and the PMO6V radiometers on VIRGO/SoHO, a re-analysis of degradation taking into account the SMM/ACRIM-I 'spin mode data' with much less exposure (see Sect. 4.2) and corrections for a glitch after the switch-off of ACRIM-I due to the repair of the SMM pointing system in 1984 that is determined by comparison with the HF and ERBS datasets as well as a proxy model. These corrections are described in more detail in Fröhlich (2006). Corrections applied to the Nimbus7/HF data include the removal of slips and glitches as discussed in Sect. 4.1, corrections for an exposure-dependent early increase, degradation and a non-exposure-dependent increase of sensitivity. In Fröhlich 2006, the corrections during the ACRIM gap are no longer determined by comparison with ERBS and a proxy model as in Fröhlich (2000), and thus, the hypothesis that the PMOD composite has been adapted according to Lean's proxy model (Scafetta 2010) is incorrect. Fröhlich applies the proxy model calibrated against the corrected ACRIM-I data only as a reference for the early observations in order to extrapolate ACRIM-I back to November 1978, the start of Nimbus7/HF. Instead, the corrections during the ACRIM gap are derived by assuming that the corresponding instruments degrade in a similar way to the PMOD like degradation. For example, the HF instrument's non-exposure-dependent increase of sensitivity is modeled in a similar way as for DIARAD/VIRGO (Fröhlich 2003). According to Fröhlich (2006), all issues related to the ACRIM gap can thus be avoided. However, that these instruments degrade similarly is a strong assumption that remains to be proven.

Clearly, the above statements do not meet the approval of Willson and Scafetta who continue to question the correctness of these modifications. Many other controversial issues between the PMOD and ACRIM advocates remain. In particular, the corrections applied by Fröhlich to the original Nimbus7 and ACRIM-I data published by the instruments' science teams have not been resolved. These points of controversy are still included in the new HF time series which is therefore continuously called into question by PMOD opponents.

\subsection{Institut Royal Météorologique Belgique Composite}

The IRMB composite (Dewitte et al. 2004) applies daily averaged TSI data from the DIARAD, PMO6V, ACRIM-II, ACRIM3, ERBS and TIM instruments. In particular, the authors use the data from PMO6V as an independent dataset, which is one of the main points of criticism brought up repeatedly against the IRMB composite.

For the construction of the IRMB composite, Dewitte et al. (2004) apply ERBE/ERBS data in order to correct the HF and ACRIM-I datasets (as in the PMOD composite), and the values adapted during this period are averages of ERBS and HF data. All datasets are referred to the space absolute radiometric reference (SARR; Crommelynck et al. 1995), 
and the adjustments for the ACRIM-II, ERBE/ERBS and VIRGO radiometers are based on direct comparison.

In the IRMB composite, no corrections are taken into account for a possible jump in the HF dataset during the ACRIM gap. This leads to a steplike rise in 1989 between the TSI of the IRMB composite and the PMOD composite (Lockwood and Fröhlich 2008). The PMOD and IRMB composites differ during the time when VIRGO/SoHO data are available (since 1996), because only the measurements of VIRGO's DIARAD instruments are used for the construction of the IRMB composite (Dewitte et al. 2004), whereas data from both types of instruments on VIRGO, the DIARAD and PMO6V instruments, are used for the construction of the PMOD composite to allow for corrections due to the non-exposuredependent increase of sensitivity of the respective instrument (Lockwood and Fröhlich 2008).

This combined approach was anticipated with the development of VIRGO (Fröhlich et al. 1995). Since the PMO6V datasets of VIRGO are ignored by Dewitte et al. (2004), the data of the IRMB composite have only been corrected for changes due to DIARAD's exposure to solar radiation, but not for DIARAD's non-exposure-dependent increase of sensitivity. In their study, Dewitte et al. (2004) identified a difference of $(+0.15 \pm 0.35) \mathrm{W} \mathrm{m}^{-2}$ between the 1986 and the 1996 activity minima. However, due to the large uncertainty of the values, this result is not statistically significant.

In an updated version of the IRMB composite, Mekaoui and Dewitte (2008) argue that the non-exposure-dependent changes of VIRGO are compensated by an averaging effect that they applied. From the comparison of the IRMB composite and a proxy regression model, they predicted that the solar minimum TSI would not change during cycle 23 with an uncertainty of $\pm 0.14 \mathrm{~W} \mathrm{~m}^{-2}$. Their conclusions were based on the assumption that, in $2008 / 2009$, the solar proxy indices would reach the same level as during the previous minimum. However, as will be discussed in the next section, this assumption turned out to be wrong during the distinct solar minimum in 2008/2009.

\section{Understanding and Modeling Solar Irradiance Variations}

Solar magnetic activity is driven by the subsurface dynamo that changes the amount, distribution and strength of the magnetic flux transported into the solar atmosphere from the convection zone below. The ultimate goal in order to understand and model solar irradiance variations is therefore to establish a physical description of the transport and eruption of magnetic flux on the Sun, as well as of the processes through which this flux changes the emission of photons from the Sun's atmosphere. In essence, this requires a complete understanding of the solar behavior.

First reports on reliable measurements of TSI changes were given in the early 1980s and were soon attributed to sunspots and faculae (e.g., Hudson et al. 1982, Willson 1982). The sunspot number is the most widely used proxy of solar activity and the one recorded for the longest time. Direct sunspot observations are available since 1610. The quantification of faculae in solar images is more difficult than that of sunspots, because of their lower contrasts in the visible spectrum and their higher fragmentation that can lead to considerable uncertainty when determining their contribution (Ortiz et al. 2002). Also, the lack of a long-term database of appropriately calibrated images makes the explicit calculation of a facular index over extended periods of time difficult. 
When sufficiently long time series of TSI data became available in the mid-1980s, the development of simple TSI reconstruction models started. The oldest (but still widely applied) irradiance models use disk-integrated proxies of solar magnetic activity, such as the sunspot number or area, plage area, the solar radio flux at $10.7 \mathrm{~cm}$, the $\mathrm{MgII}$ index or the Ca II K line (e.g., Foukal and Lean 1986, 1988). The success of these TSI models in reproducing the measured TSI variations on timescales from days to years motivated the development of more complex semiempirical models, such as the SATIRE (Spectral And Total Irradiance REconstructions) models (Fligge et al. 2000; Solanki et al. 2005; Krivova et al. 2003). These models consider contributions of different brightness structures to the irradiance change separately and require detailed radiative transfer calculations that are expensive in terms of computing time (e.g., Vögler et al. 2005). For more details and an overview of the various TSI models, see Ermolli et al. (2013), Domingo et al. (2009) or the climate review of Gray et al. (2010).

With the latest developments and achievements on the modeling side, detailed comparisons between the models and the various TSI composites have become feasible. The recent minimum of 2008/9, which was characterized by unusually long periods without sunspots, is still causing debates among the various TSI representatives. The TSI value of the 2008/2009 minimum in the PMOD composite is found to be $\sim 25 \%$ lower in terms of the mean cycle amplitude compared to the previous minimum in 1996/7 (Fröhlich 2009). However, most other solar proxy indices show much smaller relative changes of cycle amplitude during that time. On the other hand, the open solar magnetic field observed at 1 AU shows a similar change of amplitude (Fröhlich 2009, 2013). The low TSI values during the solar minimum of 2008/2009 are attributed to a change in global temperature of the photosphere that affects the UV irradiance in a different way than surface magnetic fields (Fröhlich 2009, 2013). In the earlier study, an observed change in the VIRGO TSI between 1996 and 2008, significant at the 1.5- $\sigma$ level, is reported (Fröhlich 2009). The given TSI values are $(1,365.45 \pm 0.10) \mathrm{W} \mathrm{m}^{-2}$ (for the 1996 minimum) and $(1365.26 \pm 0.16) \mathrm{W} \mathrm{m}^{-2}$ (for the 2008 minimum), respectively. However, in the 2013 review paper, no data uncertainties are included (Fröhlich 2013), neither for the activity proxies that are used nor for the reported solar cycle amplitude variations. This omission limits the assessment of the significance of the results presented.

By comparing his model results to the PMOD composite, Steinhilber (2010) was able to reproduce the 1996-2004 TSI observations. However, since the observed decrease in TSI during the 2008/2009 minimum could not be reproduced by the model, it was concluded that the observed long-term trend does not result from evolving solar surface magnetism. Instead, the difference between modeled and observed TSI was attributed to either the underrepresented weak magnetic fields in the MDI synoptic charts (used to extract the filling factors of sunspots, faculae and network), other uncertainties in the TSI measurements or a drop of the global temperature of the photosphere during the last solar cycle.

Later on, a study by Ball et al. (2012) showed that changes in the photospheric magnetic flux can reproduce almost all solar irradiance variations over the last three solar cycles surprisingly well. Their TSI-S model (where "S" indicates the satellite era) "mimics" a decline in TSI of $0.20_{-0.09}^{+0.12} \mathrm{~W} \mathrm{~m}^{-2}$ between the 1996/1997 and 2008/2009 minima. However, since this slight difference between the minima in 1996 and 2008 lies within the uncertainty range of the model, a constant TSI level for the minima cannot be ruled out either. The model is based on Kitt Peak/Spectropolarimeter (KP/SPM) and SoHO/MDI continuum images and magnetograms and is found to agree best with the PMOD composite on timescales longer than the rotational timescale. 
Krivova et al. (2011) extended the analysis of Steinhilber (2010) by comparing the SATIRE-S model output (based on Michelson Doppler Imager (MDI) magnetograms) with different TSI time series. They suggest that early degradation trends in the PMO6V radiometers on VIRGO may not have been fully accounted for in the PMOD composite leading to an overestimation of the level of TSI during the activity minimum in 1996 by roughly $0.2 \mathrm{~W} \mathrm{~m}^{-2}$ and the apparently different behavior over cycle 23 . In contrast to Fröhlich (2009) and Steinhilber (2010), they conclude that the TSI variations over cycle 23 and the change in the TSI levels between the minima in 1996 and 2008 are consistent with the solar surface magnetism mechanism. However, MDI data have been recalibrated since taking into account a position-dependent correction that has led to an increase in observed magnetic flux (Ball et al. 2011, 2012). Based on the SATIRE-S TSI reconstructions using data from either SoHO/MDI or KP/SPM, Ball et al. (2012) found that the magnetic flux in SoHO/MDI magnetograms differs before and after the period when SoHO was not in operation in 1998, which is possibly due to a focus change of the instrument. They point out that MDI magnetograms should not be used to test secular TSI variations without correcting for this effect. This inconsistency in the MDI data during the beginning of cycle 23 is shown to be almost identical to the trend in the PMOD composite during the rising phase of cycle 23 (Ball et al. 2012). This is in line with the findings of Krivova et al. (2011) who propose that the degradation correction of VIRGO during the initial years of observation (1996-1998) may not be entirely adequate.

\section{Concluding Discussion and Outlook}

A conclusive TSI time series is not only desirable from the perspective of the scientific community, but also when considering the rising interest of the public in questions related to climate change issues, thus preventing climate skeptics from taking advantage of these discrepancies within the TSI community by, e.g., putting forth a presumed solar effect as an excuse for inaction on anthropogenic warming.

Measurement continuity of the 35-year-long TSI record is essential for detecting potential long-term solar fluctuations, and it is important that these measurements continue in the future without interruptions. Offsets due to calibration differences between the instruments generally exceed the stated instrument uncertainties, and long-lasting controversial debates among the representatives of the respective TSI composites (PMOD, ACRIM, IRMB) on the cross-calibration and cross-validation of the independent observations have prevented the TSI community from coming up with a conclusive TSI composite since the first TSI composite became available in the late 1990s. Nevertheless, in recent years, some major steps forward have been taken in this process, including improved measurement accuracy and a better understanding of the causes of the offsets.

In this paper, an independent view on the studies related to the construction of such a TSI composite is presented. The main problems that have been identified include the assumption and correction of effects that have not been verified by the instrument teams, reference to work that has never been published, inappropriate use of models (and instrument data) to support results and the omission of measurement uncertainties preventing an evaluation of the validity of the results presented.

Further efforts are required to understand and quantify instrument accuracies for the instruments measuring TSI. Such work was performed during the NIST Workshop held in 2005 which focused on understanding possible reasons for this difference through an 
examination of the instrument designs and calibration approaches and helped to motivate the establishment of the TRF which, on the other hand, initiated a series of instrument calibration studies. Such instrument calibration studies need to be performed for all TSI instruments available.

Today's TSI models are not only successful in reproducing the TSI measurements, but these models have also proven to be a valuable tool for detecting and analyzing instrumental inconsistencies in the data. Nevertheless, we need to keep in mind that the true nature of solar variability lies in the magnetic field of the Sun itself. Proxies of solar activity, such as the sunspot number or area, plage area, the solar radio flux at $10.7 \mathrm{~cm}$, the $\mathrm{MgII}$ index or the Ca II K line, which all TSI models rely on, might reflect only part of the truth.

Using the individual instrument records rather than any existing TSI composite and combining the data in a new way, e.g., by applying statistical methods rather than applying corrections susceptible to subjective interpretation is a promising way to come up with a conclusive TSI composite and is one of the goals of the ongoing ISSI International Team on the "Assessment of the Accuracies and Uncertainties in the TSI Climate Data Record" led by G. Kopp ${ }^{4}$. The team includes the principal investigators of all current space-based TSI instruments as well as leading experts in the field who aim at producing an irradiance composite record having time-dependent uncertainties based on the most recent scientific results. This new solar irradiance measurement record is expected to benefit climate researchers by producing a 35-year-long time series with improved accuracy and stability.

Acknowledgments I would like to thank R.-M. Bonnet for motivating this work as well as for his advice and support in the course of its development. I would also like to thank C. Fröhlich for the illuminating discussions on the PMOD composite and S. Dewitte for providing the most recent data for the IRMB composite. Special thanks to G. Kopp and the ISSI Team "Assessment of the Accuracies and Uncertainties in the TSI Climate Data Record" for offering me the opportunity to join the discussions of the solar irradiance community. I am grateful for the helpful comments and suggestions made by the referees, one of whom was G. Kopp, during the preparation of this paper, which substantially improved its quality.

\section{References}

Abbot CG (1958) The constancy of the solar constant. Smithson Contrib Astrophys 3:13

Anklin M, Fröhlich C, Finsterle W, Crommelynck DA, Dewitte S (1998) Assessment of degradation of VIRGO radiometers on board SOHO. Metrologia 35:685-688

Ball WT, Unruh YC, Krivova NA, Solanki SK, Harder JW (2011) Solar irradiance variability: a 6-year comparison between SORCE observations and the SATIRE model. Astron Astrophys 530:A71

Ball WT, Unruh YC, Krivova NA, Solanki S, Wenzler T, Mortlock DJ, Jaffe AH (2012) Reconstruction of total solar irradiance 1974-2009. Astron Astrophys 541:A27

Butler JJ, Johnson BC, Rice JP, Shirley EL, Barnes RA (2008) Sources of differences in on-orbital total solar irradiance measurements and description of a proposed laboratory intercomparison. J Res Natl Inst Stand Technol 113:187-203

Chapman G, Cookson A, Dobias J (1996) Variations in total solar irradiance during solar cycle 22. J Geophys Res 101:13541-13548

Crommelynck D, Fichot A, Lee RB III, Romero J (1995) First realisation of the space absolute radiometric reference (SARR) during the ATLAS 2 flight period. Adv Space Res 16:817-823

Dewitte S, Crommelynck D, Mekaoui S, Joukoff A (2004) Measurement and uncertainty of the long-term total solar irradiance trend. Solar Phys 224:209

Domingo V, Ermolli I, Fox P, Fröhlich C, Haberreiter M, Krivova N, Kopp G, Schmutz W, Solanki SK, Spruit HC, Unruh Y, Vögler A (2009) Solar surface magnetism and irradiance on time scales from days to the 11-year cycle. Space Sci Rev 145(3-4):337-380

\footnotetext{
${ }^{4}$ http://www.issibern.ch/teams/solarirradiance/.
} 
Ermolli I, Matthes K, Dudok de Wit T, Krivova NA, Tourpali K, Weber M, Unruh YC, Gray L, Langematz U, Pilewskie P, Rozanov E, Schmutz W, Shapiro A, Solanki SK, Woods TN (2013) Recent variability of the solar spectral irradiance and its impact on climate modelling. Atmos Chem Phys 13(8):3945-3977

Fligge M, Solanki SK, Unruh YC (2000) Modelling irradiance variations from the surface distribution of the solar magnetic field. Astron Astrophys 353:380-388

Foukal P, Lean J (1986) The influence of faculae on total solar irradiance and luminosity. ApJ 302:826-835

Foukal P, Lean J (1988) Magnetic modulation of solar luminosity by photospheric activity. AJ 328:347-357

Fröhlich C (2000) Observations of irradiance variations. Space Sci Rev 94:15-24

Fröhlich C (2003) Long-term behaviour of space radiometers. Metrologia 40:60

Fröhlich C (2004) Solar Irradiance Variability. In: Pap JM, Fox P, Fröhlich C, Hudson HS, Kuhn J, McCormack J, North G, Sprigg W, Wu ST (eds) Solar Variability and its Effects on Climate. Geophysical Monograph 141, Washington DC American Geophysical Union Geophysical Monograph Series, vol 141, p 97

Fröhlich C (2006) Solar irradiance variability since 1978: revision of the PMOD composite during solar cycle 21. Space Sci Rev 125:53-65

Fröhlich C (2009) Evidence of a long-term trend in total solar irradiance. Astron Astrophys 501:L27

Fröhlich C (2013) Total solar irradiance: what have we learned from the last three cycles and the recent minimum? Space Sci Rev 176(1-4):237-252

Fröhlich C, Lean J (1998a) The sun's total irradiance: cycles, trends and related climate change uncertainties since 1976. Geophys Res Lett 25:4377-4380

Fröhlich C, Lean J (1998b) Total solar irradiance variations: the construction of a composite and its comparison with models. Kluwer Academic Publ, Dordrecht

Fröhlich C, Lean J (2002) Solar irradiance variability and climate. Astron Nachr 323:203-212

Fröhlich C, Romero J, Roth H, Wehrli C, Andersen BN, Appourchaux T, Domingo V, Telljohann U, Berthomieu G, Delache P, Provost J, Toutain T, Crommelynck DA, Chevalier A, Fichot A, Däppen W, Gough D, Hoeksema T, Jiménez A, Gómez MF, Herreros JM, Cortés TR, Jones AR, Pap JM, Willson RC (1995) VIRGO: experiment for helioseismology and solar irradiance monitoring. Solar Phys 162(1):101-128

Fröhlich C, Dominique A, Crommelynck DA, Wehrli C, Anklin M, Dewitte S, A F, Finsterle W, Jiménez A, Chevalier A, Roth H (1997) In-flight performance of the VIRGO solar irradiance instruments on SOHO. Solar Phys 175(2):267-286

Gray LJ, Beer J, Geller M, Haigh JD, Lockwood M, Matthes K, Cubasch U, Fleitmann D, Harrison G, Hood L, Luterbacher J, Meehl GA, Shindell D, van Geel B, White W (2010) Solar influences on climate. Rev Geophy 48(4):4001

Hoyt DV, Kyle HL, Hickey JR, Maschhoff RH (1992) The NIMBUS 7 solar total irradiance: a new algorithm for its derivation. J Geophys Res 97:51-63

Hudson HS, Silva S, Woodard M, Willson RC (1982) The effects of sunspots on solar irradiance. Solar Phys 76:211-219

Kopp G, Lean J (2011) A new, lower value of total solar irradiance: evidence and climate significance. Geophys Res Lett 38(L01):706

Kopp G, Lawrence G, Rottman G (2003) Total solar irradiance monitor design and on-orbit functionality. SPIE Proc 5171:14-25

Kopp G, Lawrence G, Rottman G (2005) The total irradiance monitor (TIM): science results. Solar Phys 230(1):129-139

Kopp G, Heuerman H, Harber D, G D (2007) The TSI radiometer facility: absolute calibrations for total solar irradiance instruments. SPIE Proc 6677:1-12

Kopp G, Fehlmann A, Finsterle W, Harber D, K H, Willson R (2012) Total solar irradiance data record accuracy and consistency improvements. Metrologia 49:S29-S33

Krivova NA, Solanki SK, Fligge M, Unruh YC (2003) Reconstruction of solar irradiance variations in cycle 23: is solar surface magnetism the cause? Astron Astrophys 399(1):L1-L4

Krivova NA, Solanki SK, Wenzler T (2009) ACRIM-gap and total solar irradiance revisited: Is there a secular trend between 1986 and 1996? Geophys Res Lett 36(20):L20101

Krivova NA, Solanki SK, Schmutz W (2011) Total solar irradiance in cycle 23. Astron Astrophys 529:A81

Lean JL, Woods TN (2010) Solar spectral irradiance: measurements and models in heliophysics. In: Schrijver JC, Siscoe GL (eds) Evolving solar activity and the climates of space and earth. Cambridge University Press, Cambridge

Lee R III, Gibson M, Wilson R, Thomas S (1995) Long-term total solar irradiance variability during sunspot cycle 22. J Geophys Res 100:1667-1675 
Lockwood M, Fröhlich C (2008) Recent oppositely directed trends in solar climate forcings and the global mean surface air temperature. II. Different reconstructions of the total solar irradiance variation and dependence on response time scale. Proc R Soc A 464:1367-1385

Mekaoui S, Dewitte S (2008) Total solar irradiance measurement and modelling during cycle 23. Solar Phys 247(1):203-216

Ortiz A, Solanki SK, Domingo V, Fligge M, Sanahuja B (2002) On the intensity contrast of solar photospheric faculae and network elements. Astron Astrophys 388(3):1036-1047

Scafetta N (2010) Climate change and its sources: a discussion about some key issues. La Chimica e IIndustria 1:70-75

Scafetta N, Willson RC (2009) ACRIM-gap and TSI trend issue resolved using a surface magnetic flux TSI proxy model. Geophys Res Lett 36(L05):701

Schmutz W, Fehlmann A, Hülsen G, Meindl P, Winkler R, Thuillier G, Blattner P, Buisson F, Egorova T, Finsterle W, Fox N, Gröbner J, Hochedez JF, Koller S, Meftah M, Meisonnier M, Nyeki S, Pfiffner D, Roth H, Rozanov E, Spescha M, Wehrli C, Werner L, Wyss JU (2009) The PREMOS/PICARD instrument calibration. Metrologia 46(4):S202-S206

Schmutz W, Fehlmann W A Finsterle, the PREMOS team (2012) First light of PREMOS/PICARD. Annual report 2011 46:44, www.pmodwrc.ch

Schmutz W, Fehlmann A, Finsterle W, Kopp G, Thuillier G (2013) Total solar irradiance measurements with PREMOS/PICARD. In: Cahalan RF, Fischer J (eds) Radiation processes in the atmosphere and ocean (IRS2012). Proceedings of the international radiation symposium (IRC/IAMAS), AIP Conf. Proc. vol 1531. AIP Publishing LLC, Melville, New York, pp 624-627

Solanki SK, Krivova NA, Wenzler T (2005) Irradiance models. Adv Space Res 35(3):376-383

Steinhilber F (2010) Total solar irradiance since 1996: is there a long-term variation unrelated to solar surface magnetic phenomena? Astron Astrophys 523:A39

Vögler A, Shelyag S, Schüssler M, Cattaneo F, Emonet T, Linde T (2005) Simulations of magnetoconvection in the solar photosphere. Astron Astrophys 429(1):335-351

Wild M, Folini D, Schär C, Loeb N, Ellsworth GD, König-Langlo G (2013) The global energy balance from a surface perspective. Clim Dyn 40:3107-3134

Willson RC (1982) Solar irradiance variations and solar activity. J Geophys Res 87:4319

Willson RC (1984) Measurements of solar total irradiance and its variability. Space Sci Rev 38:203-242

Willson RC (1994) Irradiance Observations of SMM, Spacelab 1, UARS and ATLAS Experiments in The Sun as a Variable Star, Solar and Stellar Irradiance Variations, Proceedings of IAU Colloquium 143. Cambridge University Press, Cambridge

Willson RC (1997) Total solar irradiance trend during solar cycles 21 and 22. Science 277:1963

Willson RC (2001) The ACRIMSAT/ACRIM III experiment: extending the precision, long-term total solar irradiance climate database. Earth Obs 13(3):14-17

Willson RC, Hudson HS (1991) The sun's luminosity over a complete solar cycle. Nature 351:42

Willson RC, Mordvinov AV (2003) Secular total solar irradiance trend during cycles 21-23. Geophys Res Lett 30:1199

Willson RC, Gulkis S, Janssen M, Hudson HS, Chapman GA (1981) Observations of solar irradiance variability. Science 211:700-702

Woods TN, Eparvier FG, Fontenla J, Harder J, Kopp G, McClintock WE, Rottman G, Smiley B, Snow M (2004) Solar irradiance variability during the October 2003 solar storm period. Geophys Res Lett 31:L10,802 